\title{
Débat : Poincaré et l'histoire de la relativité
}

\author{
André Rougé ${ }^{1}$ et Olivier Darrigol ${ }^{2}$ \\ (1) Laboratoire Leprince-Ringuet, École polytechnique. (2) Rehseis, Université de Paris 7, CNRS
}

\begin{abstract}
La publication de l'article d'Olivier Darrigol « Faut-il réviser l'histoire de la relativité ? » dans le Bulletin SFP $n^{\circ} 150$ de juillet-août 2005 (pp. 27-29) a suscité quelques réactions, relatives en particulier au rôle d'Henri Poincaré. Dans un souci d'objectivité, nous publions ci-dessous, sous forme d'un débat sur ce sujet, un article d'André Rougé suivi de la réponse d'Olivier Darrigol.
\end{abstract}

\section{André Rougé :}

La rédaction

Une polémique absurde sur les origines de la relativité ne doit pas faire oublier que l'apport principal de Poincaré à cette théorie n'est pas une influence éventuelle sur les premiers articles d'Einstein, mais l'introduction des notions de covariance et de groupe de symétrie. Grâce aux travaux de mathématiciens et de physiciens dont les plus connus sont Emmy Noether, Weyl et Wigner, ces idées ont façonné la physique théorique du vingtième siècle.

Le Bulletin de la SFP s'est inquiété, dans ses derniers numéros, de la parution d'un certain nombre d'ouvrages tendant à réviser l'histoire de la relativité et en attribuer la paternité à Poincaré. La polémique n'est pas nouvelle et l'on connaît la réaction d'Einstein dans des circonstances analogues : "Après tout rien ne m'oblige à lire ce machin. " La SFP n'a pas suivi l'exemple d'Einstein et semble avoir décidé de répondre à ces publications. II est important, si l'on adopte cette position, de peser soigneusement les arguments employés et, en particulier, de refuser le cadre d'un débat qui ferait d'Einstein et Poincaré deux concurrents dans une compétition dont le but est la relativité restreinte. L'accepter reviendrait à faire de l'année 1905 une fin et non le commencement dont nous célébrons le centenaire. La relation entre Einstein et Poincaré est, en vérité, tout autre : c'est celle de deux scientifiques, aux identités et projets professionnels très différents, qui apportent leurs contributions - de premier plan l'une et l'autre - à une œuvre collective.

C'est un fait historique que l'influence directe de Poincaré sur la physique relativiste de son époque a été faible. L'originalité de son apport n'a été vraiment reconnue que cinquante ans plus tard ; non pas par Whittaker, qui a surtout contribué à brouiller l'image de Poincaré en raison des réactions, parfois malhonnêtes ${ }^{1}$, qu'il a suscitées, mais par Wigner, Bargmann et Wightman quand ils ont introduit le terme « groupe de Poincaré » pour désigner le groupe de Lorentz inhomogène. II est naturel, en conséquence, d'attacher prioritairement le nom d'Einstein à la relativité restreinte, mais il ne faut pas, pour autant, minimiser et déformer la contribution de Poincaré. Cela conduirait à oublier un pan entier de la physique du vingtième siècle et le rôle qu'y ont joué un certain nombre de mathématiciens et physiciens mathématiciens, de Poincaré à Wigner en passant par Emmy Noether, Weyl et bien d'autres.

L'article d'Olivier Darrigol, publié dans le Bulletin de la SFP du mois de juillet 2005, me semble une illustration de ce risque. Après une excellente première partie, où il retrace, en historien, les apports des uns et des autres jusqu'à 1905, il remplace la suite de l'histoire par une comparaison d'Einstein et de Poincaré sur la base de critères philosophiques (épistémologie, conceptions de l'espace et du temps) qui peut donner l'impression qu'il entre dans la polémique, et trace le portrait d'un physicien croyant fermement à l'éther et n'admettant pas l'équivalence de tous les référentiels galiléens, dans lequel on reconnaît Lorentz beaucoup plus que Poincaré.

1. Cette remarque vise la publication, peu de temps après la mort d'Einstein, d'un extrait trafiqué d'une de ses lettres à partir duquel un certain nombre de commentateurs ont forgé les mythes d'un Poincaré antirelativiste et d'une animosité entre Einstein et lui.
Si le lecteur d'un des ouvrages évoqués demande à un physicien son avis sur l'origine de la relativité, il n'attend certainement pas de lui une discussion philosophique. II considérerait cela comme une dérobade. De plus, si l'intérêt d'Einstein pour la philosophie était grand, cet intérêt ne l'a jamais conduit à la stricte adhérence à un système épistémologique. II l'écrit très clairement dans sa réponse à Reichenbach, se qualifiant lui-même d'« opportuniste épistémologique ». Par ailleurs, dans la période de création de la relativité restreinte (1905-1911), les conceptions de l'espace et du temps d'Einstein étaient très proches de celles de Poincaré. Ce n'est qu'à partir de 1910 qu'il a commencé à assimiler et faire siennes les idées de Minkowski.

Quand au portrait de Poincaré, il ne résiste pas à la lecture de son œuvre, si l'on fait abstraction de sa pédagogie singulière et s'attache à en dégager le sens plutôt que de monter en épingle l'usage de certaines formulations démodées. Comme le dit fort justement Darrigol, l'éther est pour Poincaré une métaphore, " une sorte de vêtement dont nous habillons le squelette " des équations. II écrit par exemple en 1908 , dans son hommage à Lord Kelvin : " Et que dire de l'éther? En France ou en Allemagne, ce n'est guère qu'un système d'équations différentielles ; pourvu que ces équations n'impliquent pas de contradiction et rendent compte des faits observés, on ne s'inquiètera pas si l'image qu'elles suggèrent est plus ou moins étrange ou insolite. " Le terme « éther " n'apparaît que très rarement dans les écrits techniques de Poincaré, et l'on peut voir, en comparant la note et le mémoire de 1905, qu'il utilise indifféremment les expressions « milieu électromagnétique " (éther) et " champ électromagnétique ", qui est celle que préconisera Einstein pour remplacer le mot " éther ». Voir dans l'emploi par Poincaré d'expressions telles que « temps vrai » la preuve qu'il admet l'existence d'un référentiel privilégié, c'est nier ce qu'il a toujours écrit sur sa conception de la physique : «Le fond [des théories physiques] c'est l'existence de certains rapports entre des objets inaccessibles. Ces rapports sont la seule vérité que nous puissions atteindre. " Le temps vrai, comme l'espace absolu, est inaccessible au physicien et relève de la métaphysique. II le réaffirme, avec une certaine emphase, dans sa conférence de Berlin, en 1910 : «Temps absolu ! Admettrions-nous que cette expression ait un sens quelconque, qu'il nous resterait quand même inaccessible pour toujours. Tout ce que nous pouvons connaître, c'est le temps local. " II faut d'ailleurs noter que l'utilisation des termes « vrai » et « apparent » pour distinguer deux systèmes de coordonnées n'est pas courante dans les textes techniques de Poincaré. Et l'équivalence de tous les référentiels galiléens est manifeste dans son cours de Sorbonne ; notamment dans la façon dont il obtient l'équation du mouvement d'un électron dans un champ électromagné- 
tique, par un argument de covariance impeccable, certainement plus « relativiste » que celui d'Einstein en 1905 : il écrit la forme la plus générale envisageable pour l'équation, dans un référentiel arbitraire, et détermine les coefficients inconnus en demandant que cette équation soit invariante sous une transformation de Lorentz infinitésimale.

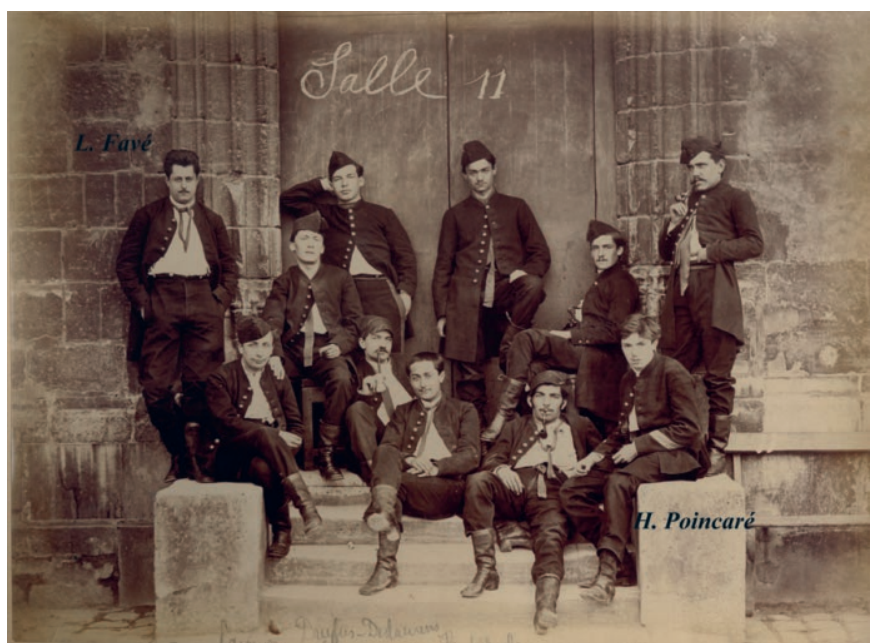

Henri Poincaré (assis à droite) pendant ses études à l'Ecole polytechnique. II s'essaya alors, avec l'aide de son camarade Favé (debout à gauche), à reproduire les expériences de Mascart qui concluaient que les phénomènes optiques sont impuissants à mettre en évidence le mouvement de translation de la Terre.

Si l'on veut comparer les apports d'Einstein et de Poincaré - ce que je ne chercherai pas à faire systématiquement - il faut avant tout tenir compte de la constatation banale qu'Einstein est un pur physicien, alors que Poincaré, qui est à l'occasion physicien, est, en premier lieu, un mathématicien. Poincaré, qui a souligné chez Einstein, dans une lettre de recommandation célèbre, la capacité de prévoir, à partir d'une idée, tous les « phénomènes nouveaux susceptibles d'être un jour vérifiés par l'expérience », ne possède pas cette capacité au même degré. Alors que, dès son premier article, Einstein étudie les conséquences expérimentales de la dilatation des temps, Poincaré semble n'avoir jamais envisagé la question. Un exemple encore plus frappant est la relation $\mathrm{E}=\mathrm{mc}^{2}$. Einstein l'obtient, en 1905, à partir d'un argument assez fragile, mais il imagine tout de suite qu'elle pourrait expliquer la production d'énergie dans la désintégration du radium. Dans son cours de Sorbonne, Poincaré étudie la conservation de la quantité de mouvement dans une collision. II établit que cette loi de conservation n'est indépendante du référentiel que si la force vive, c'est-à-dire : $\Sigma \mathrm{m} / \sqrt{ }\left(1-\mathrm{v}^{2} / \mathrm{c}^{2}\right)$, est conservée dans la collision. II utilise cette conclusion pour donner un argument supplémentaire contre une théorie poussiéreuse de la gravitation, mais il n'a pas l'idée que le résultat pourrait aussi être utile pour comprendre la radioactivité.

En revanche, cette différence professionnelle est à l'avantage de Poincaré dès que des notions mathématiques entrent en jeu, mais, pour bien le comprendre, il faut réaliser qu'il n'accorde pas toujours exactement le même sens qu'Einstein à un terme donné. De ce point de vue, le mot qui a suscité le plus d'interprétations erronées est certainement le mot "groupe ». Ceux qui affirment qu'Einstein ne connaissait pas le terme et que son emploi prouve qu'il copie Poincaré, montrent seulement qu'eux-mêmes n'ont pas lu Poincaré. On sait, par les références qu'il y a faites et par le témoignage de Solovine, qu'Einstein a lu La Science et l'Hypothèse, où le mot " groupe » apparaît au moins vingt fois. Mais, quand Einstein écrit en 1905 : " ces transformations parallèles forment, comme il se doit, un groupe ", il fait simplement la remarque banale - et présentée comme telle - que, s'il existe une transformation qui fait passer du référentiel 1 au référentiel 2 et une autre qui fait passer du référentiel 2 au référentiel 3 , il doit exister une transformation qui fait passer du référentiel 1 au référentiel 3 , transformation dont la vitesse résulte de la composition des deux premières. Par contre, quand Poincaré écrit dans sa note de 1905 : "l'ensemble de toutes ces transformations, joint à l'ensemble de toutes les rotations de l'espace doit former un groupe ", il veut dire un groupe de Lie, dont il écrit l'algèbre, et dont il sait que l'action est déterminée par celle de ses transformations infinitésimales, c'est-à-dire, en termes physiques, la théorie au premier ordre, formulée par Lorentz en 1895. C'est dire que cette seule phrase suffit à fonder l'approche " cinématique » de la relativité par Poincaré ; il n'a besoin que de vérifier la compatibilité de ce groupe avec la définition opérationnelle des grandeurs : synchronisation des horloges par l'échange de signaux lumineux et mesure des distances par le temps que la lumière met à les parcourir. Poincaré redéfinit ainsi le principe de relativité comme un principe de symétrie : l'invariance des lois physiques sous le groupe de Lorentz. Ceci est particulièrement clair dans son cours où, après l'argument de covariance mentionné plus haut, il écrit, pour introduire la description de modèles spécifiques de l'électron : "Nous avons vu une première origine de la dynamique de l'électron : le principe de relativité. On est arrivé à cette théorie par une autre voie... » Cette formulation, qui est la formulation moderne de la relativité restreinte, ne pouvait être comprise à l'époque que par des mathématiciens comme Klein ou Minkowski (qui pourtant n'utilise jamais de transformation infinitésimale). Pour les physiciens ${ }^{2}$, même les mieux formés en mathématiques, la théorie de Lie n'était pas familière et la fondation de la relativité par Einstein, à partir de deux principes et à l'aide d'expériences de pensée et de mathématiques élémentaires, possédait, indépendamment de toute autre considération, une immense supériorité pédagogique. II existe, bien sûr, de nombreuses autres raisons pour expliquer l'influence limitée des conceptions relativistes de Poincaré sur les physiciens de son temps, qui tiennent autant à l'état de la physique théorique française de son époque qu'à son mode d'exposition.

La relativité restreinte a été construite, entre 1905 et le début des années 1910, à partir de l'article "fondateur » d'Einstein, par un ensemble de scientifiques de langue allemande. Par son rôle, non seulement d'initiateur, mais aussi de pilote de cette entreprise, Einstein mérite incontestablement le titre de « père de la relativité ». La contribution indirecte de Poincaré à cette construction a été double. II a d'abord, par la Science et l'Hypothèse et, probablement, son article du Festschrift Lorentz, aidé Einstein à formuler sa conception opérationnelle du temps et réaliser l'importance du principe de relativité. II a ensuite, à travers Minkowski, fourni plusieurs éléments essentiels de la théorie : groupe de Lorentz, quadrivecteurs, etc. On peut noter que les ouvrages de référence publiés en Allemagne dans les années 20, reconnaissent de façon tout à fait correcte cette double contribution, que ce soit l'encyclopédie éditée par Teubner, avec des articles de Pauli et de Kottler, ou le Handbuch der Physik, dont le chapitre sur la relativité est rédigé par Thirring.

Plutôt que d'essayer de récrire l'histoire, les admirateurs sincères de Poincaré devraient mettre en avant l'originalité et la profondeur de sa vision des relations entre Physique et Mathématiques : "La Physique ne peut se passer des Mathématiques qui lui fournissent la seule langue qu'elle puisse parler, [et les travaux des mathématiciens] ont été d'autant plus féconds pour la physique qu'ils ont été plus exclusivement cultivés pour leur beauté propre. " Mais aussi : "Les

\footnotetext{
2. À l'exception notable de Sommerfeld - ancien collaborateur et élève de Klei - qui écrit dans son cours de physique théorique : "Le chemin suivi par Einstein en 1905 dans la découverte de la relativité restreinte était raide et difficile. [... Celui que nous prendrons est large et sans effort. [...] Un mathématicien aux yeux formés par le programme d'Erlangen de Klein aurait pu lire dans la forme des équations de Maxwell leur groupe de transformation avec toutes ses conséquences cinématiques et optiques. "
} 
Mathématiques sont quelquefois une gêne, ou même un danger quand, par la précision même de leur langage, elles nous amènent à affirmer plus que nous ne savons. Ceux qui ont cet instinct dont je vous parle savent mieux s'en servir. Ils n'y voient qu'un moyen de mieux exprimer cette symétrie qu'ils sentent dans les choses. " Cet instinct, qu'il souligne chez Curie, à propos duquel ce texte a été écrit, comme il l'a relevé antérieurement chez Maxwell, Poincaré le possède aussi, et il maîtrise, de surcroît, mieux qu'aucun de ses contemporains, l'outil mathématique nécessaire pour le mettre en œuvre : la théorie des groupes. C'est la notion de groupe de symétrie qui fait l'originalité de l'approche de la relativité par Poincaré et qui, au-delà, concourra à façonner la physique théorique du vingtième siècle. En l'associant aux principes variationnels, dont Poincaré a souligné l'importance pour l'unité de la physique, Emmy Noether établira la relation fondamentale entre invariances et lois de conservations, qui contribue en particulier, à résoudre la " crise des principes ", évoquée par Poincaré dans sa conférence de Saint-Louis. En l'introduisant dans la mécanique quantique, Weyl et Wigner écriront des chapitres essentiels de la physique moderne.

\section{Références}

A. Einstein, M. Born, Correspondance, Le Seuil (1972).

O. Darrigol, Bulletin de la SFP, 150 (2005) 27.

P.-A. Schilpp éd., Albert Einstein Philosopher-Scientist, Open Court (1949).

A. Einstein, CEuvres choisies, éd. F. Balibar et al., Le Seuil (1993).

$\mathrm{H}$. Poincaré, "Analyse de ses travaux scientifiques ", Acta Mathematica 38 (1921) 3.

H. Poincaré, Savants et écrivains, Flammarion (1910).

$\mathrm{H}$. Poincaré, La mécanique nouvelle, conférence mémoire et note sur la théorie de la relativité, Gauthier-Villars 1924, réimpression J. Gabay (1989).

H. Poincaré, Die neue Mechanik, Teubner (1911).

H. Poincaré, "Les limites de la loi de Newton, cours de Sorbonne 1906-1907 ", édité par J. Chazy, Bulletin astronomique, 17 (1953) 121, chapitres XI et XIII.

H. Poincaré, La dynamique de l'électron, conférences faites à l'école supérieure des postes et télégraphes, A. Dumas (1913).

A. Sommerfeld, Electrodynamics, Academic Press (1952).

\section{Réponse d'Olivier Darrigol à l'article d'André Rougé}

André Rougé a raison de souligner que la théorie de la relativité de Poincaré se distingue par un usage savant et novateur de la structure de groupe de Lie. ${ }^{3}$ Loin de moi la pensée que la genèse de la relativité se résume à une confrontation de systèmes philosophiques. La considération de structures formelles y joue un rôle capital, chez Einstein comme chez Poincaré. II n'en reste pas moins que l'originalité de ces deux auteurs dans l'approche de l'électrodynamique des corps en mouvement est en bonne partie liée à une critique philosophique des fondements des théories contemporaines.

André Rougé fait bien de rappeler que l'éther de Poincaré n'a rien des milieux mécaniques bien concrets imaginés par certains physiciens du XIX ${ }^{e}$ siècle. En particulier, le principe de relativité exclut tout effet détectable du vent d'éther. Néanmoins, ce milieu continue de jouer un rôle dans les raisonnements de Poincaré, y compris ceux qui s'adressent à un public averti de physiciens et de mathématiciens (voir l'appendice 1). L'architecture conceptuelle de sa théorie en dépend. Pour lui, la vitesse de la lumière n'est vraiment isotrope que dans le référentiel de l'éther et les horloges optiquement synchronisées ne donnent le temps vrai que dans ce

3. À ce sujet, voir aussi Michel le Bellac, "Le groupe de Poincaré ", à paraître dans L'héritage de Poincaré, édité par Annick Lesne, Etienne Ghys et Eric Charpentier (Belin, Paris, 2006). référentiel. Dans les autres référentiels inertiels, le temps de telles horloges n'est que «le temps apparent. » 4

Bien qu'elle soit purement conventionnelle et qu'elle soit vide de contenu empirique, cette distinction est importante pour Poincaré car elle lui permet de rester fidèle aux concepts reçus d'espace et de temps, alors qu'Einstein introduit une nouvelle cinématique et Minkowski une nouvelle géométrie de l'espace-temps. Poincaré s'est explicitement distancié de telles tentatives : «Aujourd'hui certains physiciens veulent adopter une convention nouvelle. Ce n'est pas qu'ils y soient contraints ; ils jugent cette convention nouvelle plus commode, voilà tout ; et ceux qui ne sont pas de cet avis peuvent légitimement conserver l'ancienne et ne pas troubler leurs vieilles habitudes. Je crois entre nous que c'est ce qu'ils feront encore longtemps. $»^{5}$

En tant que physicien, André Rougé juge l'éther de Poincaré comme décoratif et secondaire et situe l'essentiel de cette théorie dans le groupe de Poincaré comme traduction du principe de relativité. L'historien que je suis n'a pas cette liberté. II doit constater que Poincaré maintenait l'éther dans sa dynamique de l'électron et ne jugeait pas utile de réformer les concepts d'espace et de temps. Et il doit se garder de prêter à Poincaré des intentions incompatibles avec ses textes et leurs contextes.

Enfin, je me réjouis de voir qu'André Rougé partage mon aversion pour les pseudo-histoires de la relativité écrites sur le mode d'une compétition posthume entre Einstein et Poincaré. Le but de l'article paru dans le Bulletin $n^{\circ} 150$ était bien de montrer que la construction de la théorie de la relativité fut un travail graduel et collectif auquel Lorentz, Einstein et Poincaré ont contribué dans des mesures comparables.

\section{Appendice 1}

\section{Quelques citations de Poincaré concernant l'éther}

(Pour être bien comprises, ces citations doivent être lues dans le contexte où elles apparaissent. Néanmoins, elles donnent une première indication que l'éther continue de jouer un rôle important dans la physique de Poincaré de 1905 à sa mort.)

Dans son article de juin 1905 et dans le mémoire de Palerme (1906), Poincaré énonce le postulat de relativité comme l'impossibilité de détecter « le mouvement absolu de la Terre ou plutôt son mouvement, non par rapport aux autres astres, mais par rapport à l'éther » (CEuvres 9, pp. 489 et 495, souligné par moi). De même, en 1908, il précise que ce qu'il appelle « vitesse absolue de la Terre » n'est pas vraiment une vitesse absolue : "II ne s'agit pas, en effet, de la vitesse par rapport à un espace absolu vide, mais par rapport à l'éther, que l'on regarde par définition comme étant en repos absolu " (CEuvres 9, p. 562).

Dans le mémoire de Palerme, Poincaré écrit encore : « Si la propagation de l'attraction [gravitationnelle] se fait avec la vitesse de la lumière, cela ne peut être par une rencontre fortuite, cela doit être parce que c'est une fonction de l'éther ; et alors il faudra chercher à pénétrer la nature de cette fonction, et la rattacher aux autres fonctions de ce fluide »(CEuvres 9, p. 497).

En 1908, Poincaré décrit la théorie des électrons de Lorentz (pour laquelle il penche) de la manière suivante : « Tous ces électrons baigneraient dans l'éther... En dehors des électrons et de l'éther il n'y aurait rien. » Un peu plus loin, il commente

4. O. Darrigol, "Henri Poincaré's criticism of fin de siècle electrodynamics", Studies in the history and philosophy of physics, 26 (1995), 1-44 ; Electrodynamics from Ampère to Einstein (Oxford, 2000); "The genesis of the theory of relativity," http://www.lpthe.jussieu.fr/poincare/textes/avril2005/darrigol.pdf, also in Poincaré seminar 2005, eds J.B. Duplanchier et al. (Basel: Birkhäuser, 2006); "The mystery of the Einstein-Poincaré connection," Isis, 95 (2004), 614-626. 5. "L'espace et le temps", conférence de Londres du 4 mai 1912, dans Dernières pensées (Paris, 1913), pp. 97-109, à la page 109. 
favorablement l'hypothèse d'une masse mécanique nulle des électrons et précise : "Dans cette hypothèse, l'inertie est d'origine exclusivement électromagnétique ; elle se réduit à l'inertie de l'éther. Les électrons ne sont plus rien par euxmêmes ; ils sont seulement des trous dans l'éther, et autour desquels s'agite l'éther; plus ces trous sont petits, plus il y aura d'éther, plus par conséquent l'inertie de l'éther sera grande » (CEuvres 9, pp. 557, 559).

Dans le même texte de 1908, Poincaré discute la synchronisation d'horloges par échange de signaux optiques et montre que cette synchronisation reste transitive quand les horloges se meuvent à la même vitesse « par rapport à l'éther immobile ", si l'on admet la contraction de Lorentz et si l'on fait comme si la propagation de la lumière était encore isotrope (pour des observateurs en mouvement). II est tout à fait clair que pour Poincaré cette procédure de synchronisation implique "un réglage défectueux " des horloges en mouvement. Celles-ci ne donnent, pour les trajets lumineux, que des « durées apparentes » proportionnelles à des " distances apparentes » (mesurées avec des règles contractées). Un peu plus loin, Poincaré montre que pour que les vitesses se composent sans que le résultat n'excède jamais la vitesse de la lumière, « il faut les compter non avec le temps vrai, mais avec le temps local [donné par la transformation de Lorentz] " (CEuvres 9, pp. 563, 564, 567, 574). Cette distinction est maintenue dans les leçons de 1912 à l'École des Postes et Télégraphes (voir le site des Archives Poincaré), peu avant la mort de Poincaré. Une de ses dernières conférences, donnée à la SFP dans la même année s'intitule « Les rapports de la matière et de l'éther » (CEuvres 9, pp. 669-682). II y est abondamment question de l'éther comme vecteur du rayonnement lumineux.

\section{Appendice 2}

\section{Textes fondateurs disponibles sur Internet}

Textes de Poincaré : Voir la section «ressources " du site des Archives Poincaré (http://www.univ-nancy2.fr/poincare/ ), l'édition Guillaume des principaux textes concernant la relativité (intitulée La mécanique nouvelle) disponible sur Gallica (http://gallica.bnf.fr/) et la conférence de Berlin Die neue Mechanik sur le site de l'université du Michigan (http://www.hti.umich.edu/u/umhistmath/).

Textes d'Einstein : Pour des traductions françaises des textes de 1905, voir le site de l'Année mondiale de la physique (http://www.physique2005.org/04_articles/00.htm). Pour les originaux allemands, voir le site de l'Université d'Augsburg (http://www.physik.uni-augsburg.de/annalen/history/Einsteinin-AdP.htm).

Textes de Lorentz : La Theory of electrons de 1906 se trouve sur Gallica (http://gallica.bnf.fr/).

Textes de Minkowski : Les principaux textes concernant la relativité (en allemand) se trouvent sur le site du projet de numérisation de l'Université de Göttingen (http://gdz.sub.uni-goettingen.de/en/index.html). Une traduction française de la conférence de Cologne a été publiée en 1909 dans les Annales scientifiques de l'ENS (http://www.numdam.org/). 